\title{
The impact of standardized methods of hepatic vein reconstruction with an external iliac vein graft
}

\author{
Fumihiro Terasaki, Yuji Kaneoka, Atsuyuki Maeda, Yuichi Takayama, Yasuyuki Fukami, \\ Takamasa Takahashi, Masahito Uji \\ Department of Surgery, Ogaki Municipal Hospital, Ogaki, Japan \\ Contributions: (I) Conception and design: F Terasaki, Y Kaneoka, Y Fukami; (II) Administrative support: F Terasaki, Y Kaneoka, Y Fukami; (III) \\ Provision of study material or patients: All authors; (IV) Collection and assembly of data: F Terasaki, Y Fukami; (V) Data analysis and interpretation: \\ F Terasaki, Y Fukami; (VI) Manuscript writing: All authors; (VII) Final approval of manuscript: All authors. \\ Correspondence to: Fumihiro Terasaki, MD. Department of Surgery, Ogaki Municipal Hospital, 4-86 Minaminokawa-cho, Ogaki, Gifu 503-8502, \\ Japan. Email: fumihiro0207@gmail.com.
}

\begin{abstract}
Background: Liver tumors that invade the hepatic vein are surgically challenging, especially in patients with liver dysfunction. Preservation of as much of the parenchyma as possible is important; thus, when feasible, we perform hepatectomy with hepatic vein reconstruction (HVR) using an external iliac vein (EIV) graft. We conducted a retrospective study to investigate the benefit of HVR and to evaluate our procedure.

Methods: The study included patients treated by hepatectomy with HVR using EIV grafts and vascular clips. We reviewed the surgical outcomes, including total operation and HVR times, postoperative complications, and postoperative liver function.

Results: The surgeries included right $\operatorname{HVR}(\mathrm{n}=13)$, left $\operatorname{HVR}(\mathrm{n}=3)$, and middle HVR $(\mathrm{n}=1)$. The total operation time was 277 \pm 72 minutes (155-400 minutes), and the HVR time was 27 \pm 5 minutes (1940 minutes). Graft patency was confirmed in 14 (82\%) of the patients. One patient who underwent HVR with running sutures required emergency surgery due to graft thrombosis. Clavien-Dindo > grade IIIa postoperative complications occurred in $4(23.5 \%)$ patients, but there were no treatment-related deaths.

Conclusions: In conclusion, our hepatic resections with HVR using the same techniques and graft materials showed acceptable surgical outcomes. From our experience, we believe that preparatory hepatic resection with HVR is an effective treatment, especially for patients with decreased liver function or with a small residual liver parenchyma.
\end{abstract}

Keywords: Hepatectomy; hepatic vein reconstruction (HVR); external iliac vein graft

Submitted Jul 20, 2019. Accepted for publication Sep 20, 2019.

doi: 10.21037/hbsn.2019.09.18

View this article at: http://dx.doi.org/10.21037/hbsn.2019.09.18

\section{Introduction}

Hepatic resection has become a fairly safe procedure, and perioperative mortality associated with hepatic resection has been reduced to less than $5 \%$ (1), but major hepatic resection is still risky, and the mortality associated with this procedure has remained unchanged over time (2). Hepatic resection can be quite challenging in patients with cirrhosis or decreased liver function; thus, an adequate hepatectomy with hepatic vein resection/reconstruction (HVR) is generally performed when a hepatic tumor invades or envelops the hepatic vein in order to achieve a negative surgical margin and to preserve the function of the remnant liver. The significance of HVR in preserving remnant liver function was first reported by Nakamura in 1990 (3) and has been confirmed in the last three decades $(4,5)$. In addition, surgical techniques derived from living donor liver transplantation, such as hepatic vascular exclusion (6), venovenous bypass using heparin-coated Anthron Tube $(7,8)$, and ex-vivo hepatic resection (9), have been developed, and HVR has since been considered an important procedure for 

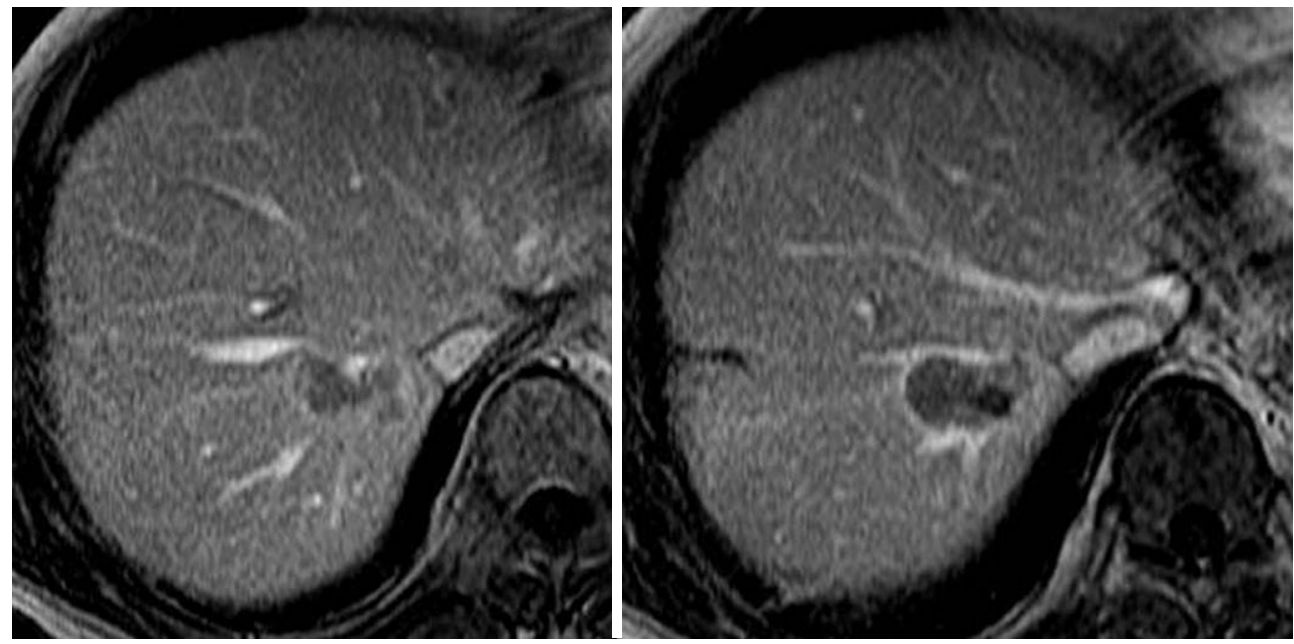

Figure 1 Magnetic resonance image showing a tumor in contact with the right hepatic vein.

the treatment of tumors involving the hepatic vein.

Only a relatively short time has passed since HVR was routinely performed as part of the hepatic resection procedure, so the optimum HVR techniques, graft selection, and potential complications have not been fully investigated. We performed hepatectomies with HVR and applied the same techniques in each patient: the use of an external iliac vein (EIV) graft and vascular clips (10). To investigate the surgical outcome of HVR, we reviewed the operative and postoperative outcomes. We present the following article in accordance with the STROBE reporting checklist (available at https://hbsn.amegroups.com/article/ view/10.21037/hbsn.2019.09.18/rc).

\section{Methods}

\section{Study patients}

This study was conducted in accordance with the Declaration of Helsinki (as revised in 2013). The study was approved by institutional ethics board of 20210325-6 and informed consent was taken from all individual participants. Between August 1996 and October 2014, consecutive patients who underwent hepatectomy with HVR for hepatocellular carcinoma (HCC) or for metastatic liver tumors were included in this study. The patients' clinical characteristics, including patient background, surgical outcomes such as hepatic vein resection and reconstruction, hepatic vein reconstruction (HVR) time, length of the vein graft, postoperative complications and postoperative liver function, were reviewed retrospectively from the patients' medical records. Graft patency was checked by computed tomography (CT), which was performed every 3 to 6 months after the surgery and continued for at least 5 years. All patients signed informed consent forms before undergoing surgery. Thirty-day postoperative complications, including mortality, were graded according to the Clavien-Dindo classification system (11).

\section{Surgical indication}

The indications for hepatectomy with HVR in our institution were as follows: (I) when the tumor was pressing on the hepatic vein or when the tumor appeared on preoperative images as surrounding more than half of the hepatic vein (Figure 1); (II) tumor involvement of a hepatic vein and resection that would result in a small remnant liver volume $(<300 \mathrm{~mL})$; (III) a possibility of repeat hepatectomy in the future; and (IV) when indocyanine green retention for at 15 minutes (ICGR15) was $>20 \%$.

\section{Surgical procedure}

The surgery in all patients began with midline and right subcostal incisions. The hepatic vein was exposed at the point where it emptied into the inferior vena cava. Hemihepatic vascular occlusion was then achieved with vessel loops in preparation for hepatectomy. Intraoperative ultrasonography (IOUS) was often used to determine the length of the hepatic vein to be resected. Clamping usually lasted 15 minutes, and the declamping period was 


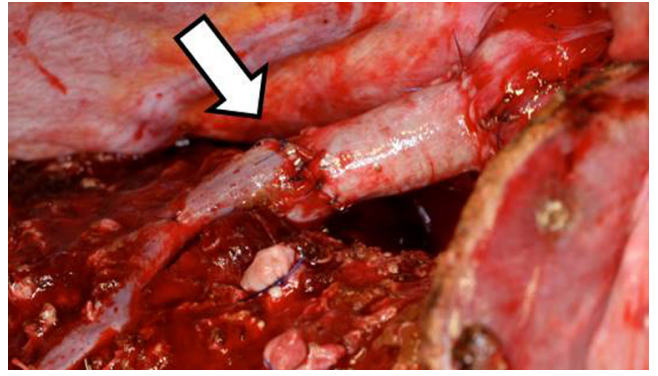

Figure 2 Vascular clips are applied during right hepatic vein reconstruction (arrow).

5 minutes. Hepatic congestion governed the timing for harvest of an EIV graft-the best time was usually just before resection of the hepatic vein (12). The graft was harvested extraperitoneally through an upper groin incision. At the peripheral stump, the graft was resected at a point more central than the point at which it was joined by the communicating branch of the obturator vein to preserve return flow from the peripheral side. The edges of the EIV were closed with 3-0 polypropylene transfixing sutures or 5-0 polypropylene running sutures. HVR was performed after extirpation of the specimen, and the graft was placed in a reverse position. At first, we attached the graft to the proximal end of the hepatic vein at 4 points with 6-0 polypropylene sutures, and afterwards, we used mediumsized vascular clips (medium-sized, United States Surgical) 4-5 times between each stich (Figure 2). In some cases, however, applying the clips was difficult, so we attached the posterior wall of the hepatic vein to the graft with a running suture of 6-0 polypropylene, and vascular clips were applied to the anterior wall. After reconstruction, IOUS was used to check blood flow and confirm triphasic waves.

\section{Perioperative anticoagulation}

We did not typically use heparin or other anti-coagulative agents during HVR. However, 5,000 U of heparin was routinely administered on postoperative day 0 after all abdominal surgeries, and 10,000 U/day of heparin was routinely administered on postoperative days 1-2 to prevent any venous thrombosis. This is not a regimen specific to HVR, and we usually do not add other medications to heparin. We tightly applied a bandage on the right leg immediately after surgery in order to prevent edema.

\section{Results}

\section{Surgical details}

This study included 17 consecutive patients with HCC $(n=11)$ or metastatic liver tumors $(n=6)$ treated by hepatectomy with HVR. The patients' details are shown in Table 1. The male/female sex ratio was $14 / 3$, and the patients ranged in age from 53 to 79 years (mean, 66.4 years). The tumor was located in segment $7 / 8$ in 13 patients, in segment 2 in 2 patients, and in segment 4 in 2 patients. The surgical outcome, graft patency, and postoperative course of HVR per patient are shown in Table 2. The following surgical procedures were performed: resection of segment $7 / 8$ with resection and reconstruction of the right hepatic vein (RHV) $(n=13)$, resection of segment 2 with resection and reconstruction of the left hepatic vein (LHV) $(n=3)$, and resection of segment $2 / 4$ with resection and reconstruction of the middle hepatic vein (MHV) ( $\mathrm{n}=1)$. The total operation time ranged from 155 to 400 minutes, with a mean \pm SD of $277 \pm 72$ minutes. The HVR time ranged from 19 to 40 minutes, with a mean \pm SD of $27 \pm 5$ minutes. Intraoperative blood loss ranged from 310 to $3,030 \mathrm{~mL}$, with a mean of $1,000 \mathrm{~mL}$. The grafts ranged in length from 2 to $4 \mathrm{~cm}$.

\section{Graft patency}

Graft patency was confirmed in 14 (82\%) of the 17 patients. Graft occlusion was observed on the CT images 2 weeks after the surgery in 2 cases (patients 10 and 12), but these patients' conditions were stable, and they were discharged without incident. Early obstruction of the graft was observed in 1 patient (patient 16), and emergency surgery was required. Two days after the first surgery, this patient's blood tests revealed elevated hepatic enzymes, leading to our decision to perform the emergency surgery. Upon surgery, we found stricture at the anastomosis and thrombotic obstruction of the entire graft (Figure $3 A$ ). We harvested part of the left EIV and attached the graft to the peripheral end of the existing graft to make up for the original graft that we discovered was too short. We sutured the new graft to the existing graft at 4 points with 6-0 polypropylene and then placed vascular clips 4-5 times between each suture. We also sutured the new graft and the remnant hepatic vein with the same method (Figure 3B). The patient was discharged 19 days after 
Table 1 Study patients and case details

\begin{tabular}{lcccccc}
\hline Patient & Sex & Age (years) & Disease & Segment(s) & ICGR15 (\%) & Primary hepatectomy \\
\hline 1 & M & 75 & HCC & S7/8 & 24.8 & - \\
2 & M & 53 & LM & S7/8 & 2.1 & - \\
3 & M & 71 & HCC & S7/8 & 16.2 & - \\
4 & M & 66 & LM & S7/8 & 3.5 & - \\
5 & F & 67 & HCC & S7/8/1r & 11.4 & - \\
6 & M & 77 & HCC & S7/8 & 8.3 & - \\
7 & M & 70 & HCC & S7/8/1r & 21 & - \\
8 & M & 67 & HCC & S7/8 & 22.9 & - \\
9 & M & 56 & HCC & S7/8 & 8 & - \\
10 & F & 70 & HCC & S7/8 & 21 & - \\
11 & M & 55 & HCC & S7/8/1r & 10.8 & - \\
12 & M & 79 & HCC & S7/8 & 11.4 & - \\
13 & M & 68 & LM & S7/8/1r & 7.5 & - \\
14 & M & 55 & LM & S2 & 8.6 & Right hemihepatectomy \\
15 & M & 72 & HCC & S2 & 23.4 & S8 partial resection \\
16 & F & 67 & LM & S4b/2/1r/11 & 17.8 & Posterior sectionectomy \\
17 & M & 60 & LM & S4b/1r & 17.2 & Posterior sectionectomy \\
\hline
\end{tabular}

HCC, hepatocellular carcinoma; LM, liver metastasis; ICGR15, indocyanine green retention in 15 minutes; 1r, right caudate lobe; 1I, left caudate lobe; 4b, superior medical segment; S8, anterior superior segment.

this second surgery. The graft remained 1 year and 4 months after left hepatic vein reconstruction (Figure 3C). Graft patency was confirmed at the last follow-up in the remaining $14(82 \%)$ patients. Graft patency was documented for 14 years and 3 months in 1 case (patient 10).

\section{Postoperative course}

Hepatic enzyme and biliary enzyme levels from day 0 to postoperative day 7 are shown for 16 of the 17 patients in Figure 4. In the 16 patients, the mean [range] preoperative aspartate aminotransferase (AST) and alanine aminotransferase (ALT) levels were 51 [15-154] IU/L and 48 [14-146] IU/L, respectively. The AST and ALT levels in the 16 patients increased postoperatively up to 577 and $451 \mathrm{IU} / \mathrm{L}$, respectively, within the first 5 days after surgery but decreased to almost normal levels by day 7 . The mean total bilirubin level was $0.8 \mathrm{mg} / \mathrm{dL}$ (range, $0.2-2.8 \mathrm{mg} / \mathrm{dL}$ ) before surgery, and it increased substantially to 2.5 within the first 3 days after surgery but then decreased to less than $1.5 \mathrm{mg} / \mathrm{dL}$ by day 7 in most cases. The AST and ALT levels in the patient who suffered early graft obstruction reached 6,264 and 2,337 IU/L, respectively, on postoperative day 2 and decreased after the emergency surgery.

The mean postoperative length of stay was 20 [14-60] days. Postoperative complications at Clavien-Dindo grade IIIa or above occurred in $4(23.5 \%)$ of the 17 patients. These complications consisted of the graft occlusion mentioned above $(n=1)$, hepatic coma $(n=1)$, bile leakage $(n=1)$, and duodenal ulcer $(n=1)$. There were no surgery-related deaths.

\section{Histologic findings}

Histologically, HCC was confirmed in 11 patients and metastatic liver tumors in 6 patients. Invasion of the tumor into the wall of a hepatic vein was found in 4 patients, and attachment to the vein was found in the remaining 13 patients. The tumor was exposed on the cut surface of the specimen in 4 of the 17 patients. 
Table 2 Outcomes of hepatic vein reconstruction, per study patient

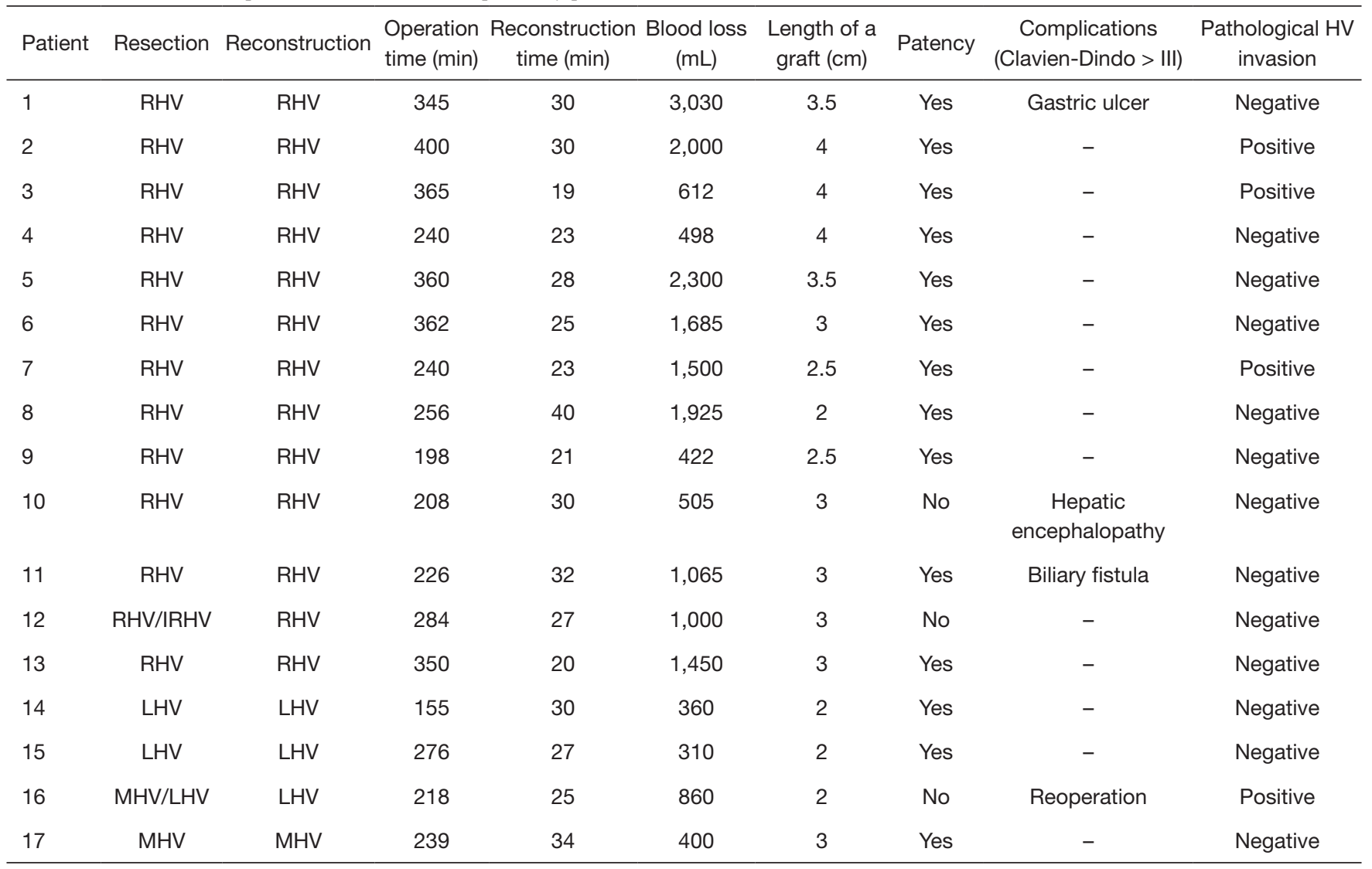

RHV, right hepatic vein; MHV, middle hepatic vein; LHV, left hepatic vein; IRHV, inferior right hepatic vein; REIV, right external iliac vein; HV, hepatic vein.

\section{Discussion}

This study showed the surgical outcome of 17 hepatic resections with HVR using the same techniques and graft materials. Although hepatic resection with HVR is thought to be a high-risk surgical technique, and there have been reports of associated operative mortality rates of approximately $11-25 \%(13,14)$, the postoperative mortality rate was $0 \%$ among our patients. Our study suggests that surgical methods, graft choice, and surgical technique can each play a role in decreasing early mortality, and preparatory hepatic resection with HVR performed with an EIV graft is an effective treatment for patients with decreased liver function or with a small residual liver parenchyma.

Early mortality after major hepatic resection has decreased to less than $5 \%(1,2)$, but major hepatic resection is still risky, and the mortality associated with this procedure has remained unchanged over time. Although palliative liver resection is considered to be the best option in patients with cirrhosis or decreased liver function, hepatectomy with HVR should be considered to obtain a tumor-free margin (15) and to prolong patient survival after liver resection.

Resection of liver tumors involving hepatic veins requires careful planning, especially in patients with liver dysfunction (16). The indications for preparatory hepatectomy with HVR are (I) tumor involvement of a hepatic vein and resection that would result in a small remnant liver volume or (II) a possibility of repeat hepatectomy. Reconstruction of the RHV is considered for resection of segment 7 or 8 or both as well as for absence of a large inferior right hepatic vein (IRHV) (17). If an IRHV exists, it is possible to resect the main hepatic veins (18), leaving segments 5 and 6 dependent on venous outflow through the IRHV or intrahepatic collaterals (5). A large IRHV that drains all of segment 6 is present in $20-24 \%$ of 

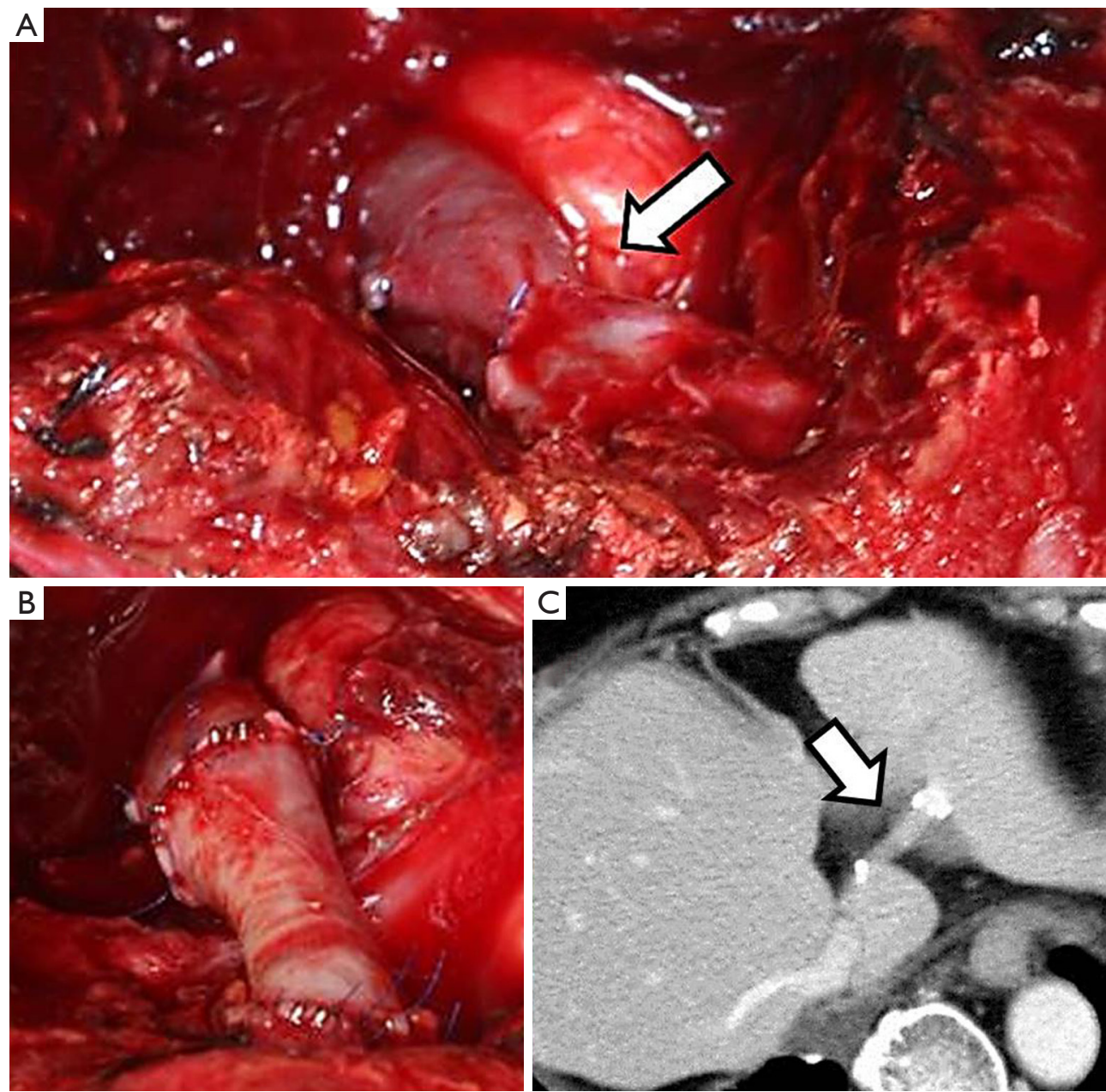

Figure 3 Surgical details in emergency surgery (A,B) and the patent graft after surgery (C). (A) In this case of left hepatic vein reconstruction, a running suture of 6-0 polypropylene was applied to both stumps. Stricture occurred at the distal stump (arrow); (B) reoperation due to graft occlusion involved extending the existing graft with a left external iliac vein graft and securing the graft with vascular clips; (C) the graft remained patent for 1 year and 4 months after left hepatic vein reconstruction (arrow).
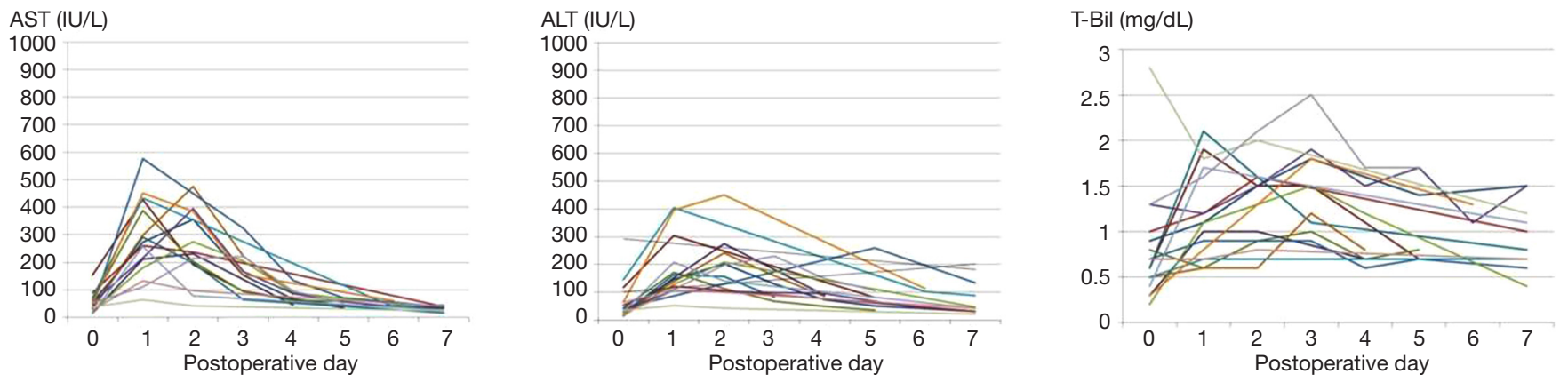

Figure 4 Line graphs showing preoperative and postoperative (over 7 days) hepatic enzyme (AST, ALT, and total bilirubin) levels in 16 patients. AST, aspartate aminotransferase; ALT, alanine aminotransferase. 
reported patients (19). A previous clinical study revealed 7 different combinations of hepatic veins draining segment 5 and segment 6 , many of which depended on the RHV as the chief drainage vein; this was the case for $82.5 \%$ of the reported patients requiring HVR (20). MHV reconstruction is considered in cases of hepatectomy involving segment 4 and the absence of a thick IRHV. LHV reconstruction is considered in cases of segmentectomy for a tumor located at the exit site of the LHV, and segment 3 should be preserved to avoid postoperative liver dysfunction (21).

We performed HVR in all 17 of our patients with the use of an EIV graft. Long-term graft patency was achieved in $14(82 \%)$ of the 17 patients. An embolism developed in $3(18 \%)$ of the 17 patients, and in one patient, complete occlusion of the graft necessitated emergency operation. In this case, both ends of the $2-\mathrm{cm}$ graft were sewn with a running suture. This is the only patient in whom we used running sutures on both ends, so we speculate that the running suture caused the stricture. A running suture can cause torsion because of the slight differences in the distance and direction of the suture pitches, an issue that is avoided when vascular clips are used. Thus, we recommend the use of vascular clips, as described by Kirsch et al. (22) in 1992. Use of vascular clips shortens the reconstruction time, prevents stricture, and can prevent vascular intimal injury and thrombosis, which usually result from the exposed threads. The clips are placed outside and not inside the graft.

The length of the graft also seems to be important. Intraoperatively, the graft in the reoperation case seemed to be of appropriate length, but in light of the hepatic congestion that ensued after abdominal closure, we realized that the graft was too short. A graft that is too short in combination with hepatic congestion inevitably causes graft occlusion because the resulting tension becomes selfperpetuating (23). To avoid this problem, we believe it is important to perform adequate hepatectomy, meaning that a negative surgical margin is obtained and ensuring that the graft is of adequate length.

Graft selection is discussed in many reports. It remains controversial whether an autologous graft or artificial graft is suitable for HVR. Several studies have shown advantages and disadvantages of artificial grafts, especially polytetrafluoroethylene (PTFE) grafts (24). The clear advantage of an artificial graft is the various lengths and diameters from which to choose. In addition, the reported risk of infectious and surgical complications is approximately $0.5 \%$ (25). However, the disadvantage of an artificial graft is the potential for long-term stricture and thrombosis. Artificial grafts may be useful in transplantation surgery, but for tumor resection, graft occlusion is of major concern with respect to remnant liver function. The clear advantage of an autologous graft is the decreased possibility of occlusion. Graft patency was documented at 14 years and 3 months in 1 of our patients, and there has since been no evidence to the contrary. For autologous grafts, the ovarian vein (26), EIV (12), and saphenous vein (3) are often used, and several studies have shown the usefulness of a peritoneal patch (27). Previous reports (12) have shown the suitability of the EIV for RHV reconstruction and of the superficial femoral vein for MHV and LHV reconstructions because these veins, compared to other veins, have considerably fewer branches. We consider the EIV graft particularly suitable for HVR because the diameter is the same as that of the hepatic vein, and we can harvest a graft that is longer than other vein grafts.

Several limitations associated with the present study warrant mention. This was a retrospective study conducted over a long period of time and due to the small number of patients, we could not perform statistical analysis for graft occlusion. It is thus necessary to accumulate further cases.

In conclusion, our hepatic resections with HVR using the same techniques and graft materials showed acceptable surgical outcomes. From our experience, we believe that preparatory hepatic resection with HVR is an effective treatment, especially for patients with decreased liver function or with a small residual liver parenchyma. The techniques we used appear to be widely applicable to cases of extensive hepatectomy.

\section{Acknowledgments}

Funding: This work described herein was supported by departmental resources.

\section{Footnote}

Reporting Checklist: The authors have completed the STROBE reporting checklist. Available at https://hbsn. amegroups.com/article/view/10.21037/hbsn.2019.09.18/rc

Data Sharing Statement: Available at https://hbsn. amegroups.com/article/view/10.21037/hbsn.2019.09.18/dss

Conflicts of Interest: All authors have completed the ICMJE uniform disclosure form (available at https://hbsn. 
amegroups.com/article/view/10.21037/hbsn.2019.09.18/coif). The authors have no conflicts of interest to declare.

Ethical Statement: The authors are accountable for all aspects of the work in ensuring that questions related to the accuracy or integrity of any part of the work are appropriately investigated and resolved. This study was conducted in accordance with the Declaration of Helsinki (as revised in 2013). The study was approved by institutional ethics board of 20210325-6 and informed consent was taken from all individual participants.

Open Access Statement: This is an Open Access article distributed in accordance with the Creative Commons Attribution-NonCommercial-NoDerivs 4.0 International License (CC BY-NC-ND 4.0), which permits the noncommercial replication and distribution of the article with the strict proviso that no changes or edits are made and the original work is properly cited (including links to both the formal publication through the relevant DOI and the license). See: https://creativecommons.org/licenses/by-nc-nd/4.0/.

\section{References}

1. Aloia TA, Fahy BN, Fischer CP, et al. Predicting poor outcome following hepatectomy: analysis of 2313 hepatectomies in the NSQIP database. HPB (Oxford) 2009;11:510-5.

2. Kingham TP, Correa-Gallego C, D'Angelica MI, et al. Hepatic parenchymal preservation surgery: decreasing morbidity and mortality rates in 4,152 resections for malignancy. J Am Coll Surg 2015;220:471-9.

3. Nakamura S, Sakaguchi S, Kitazawa T, et al. Hepatic vein reconstruction for preserving remnant liver function. Arch Surg 1990;125:1455-9.

4. Kaneoka Y, Yamaguchi A, Isogai M, et al. Preparatory hepatic resection with right hepatic vein reconstruction for paracaval liver tumor. J Hepatobiliary Pancreat Surg 2002;9:265-70.

5. Hemming AW, Reed AI, Langham MR, et al. Hepatic vein reconstruction for resection of hepatic tumors. Ann Surg 2002;235:850-8.

6. Huguet C, Addario-Chieco P, Gavelli A, et al. Technique of hepatic vascular exclusion for extensive liver resection. Am J Surg 1992;163:602-5.

7. Shaw BW, Jr., Martin DJ, Marquez JM, et al. Venous bypass in clinical liver transplantation. Ann Surg 1984;200:524-34.
8. Takayama T, Nakatsuka T, Yamamoto J, et al. Rereconstruction of a single remnant hepatic vein. Br J Surg 1996;83:762-3.

9. Hemming AW, Chari RS, Cattral MS. Ex vivo liver resection. Can J Surg 2000;43:222-4.

10. Kaneoka Y, Yamaguchi A, Isogai M, et al. Hepatic vein reconstruction by external iliac vein graft using vascular clips. World J Surg 2000;24:377-82.

11. Clavien PA, Barkun J, de Oliveira ML, et al. The ClavienDindo classification of surgical complications: five-year experience. Ann Surg 2009;250:187-96.

12. Kaneoka Y, Maeda A, Isogai M. Surgical outcome of autologous external iliac vein grafting in cases of hepatopancreato-biliary malignancy: how I do it. J Gastrointest Surg 2012;16:1590-6.

13. Lodge JP, Ammori BJ, Prasad KR, et al. Ex vivo and in situ resection of inferior vena cava with hepatectomy for colorectal metastases. Ann Surg 2000;231:471-9.

14. Madariaga JR, Fung J, Gutierrez J, et al. Liver resection combined with excision of vena cava. J Am Coll Surg 2000;191:244-50.

15. Zhong FP, Zhang YJ, Liu Y, et al. Prognostic impact of surgical margin in patients with hepatocellular carcinoma: A meta-analysis. Medicine (Baltimore) 2017;96:e8043.

16. Das BC, Isaji S, Kawarada Y. Analysis of 100 consecutive hepatectomies: risk factors in patients with liver cirrhosis or obstructive jaundice. World J Surg 2001;25:266-72; discussion 272-3.

17. Makuuchi M, Hasegawa H, Yamazaki S, et al. Four new hepatectomy procedures for resection of the right hepatic vein and preservation of the inferior right hepatic vein. Surg Gynecol Obstet 1987;164:68-72.

18. Superina RA, Bambini D, Filler RM, et al. A new technique for resecting 'unresectable' liver tumors. J Pediatr Surg 2000;35:1294-9.

19. Couinaud C. Études anatomicales et chirurgicales. Paris: Masson, 1957.

20. Togo S, Shizawa R, Kanemura E, et al. Indications for posthepatectomy hepatic vein reconstruction from functional and morphological studies: clamping test and hepatic vein branch distribution determined by three-dimensional computed tomography. Hepatogastroenterology 2001;48:655-9.

21. Nakamura S, Sakaguchi S, Hachiya T, et al. Significance of hepatic vein reconstruction in hepatectomy. Surgery 1993;114:59-64.

22. Kirsch WM, Zhu YH, Hardesty RA, et al. A new method 
for microvascular anastomosis: report of experimental and clinical research. Am Surg 1992;58:722-7.

23. Parmaksizoglu F, Beyzadeoglu T. A modified method of microvascular autogenous interposition vein grafting for vascular reconstruction. J Reconstr Microsurg 2002;18:191-4.

24. Orimo T, Kamiyama T, Yokoo H, et al. Usefulness of artificial vascular graft for venous reconstruction in liver surgery. World J Surg Oncol 2014;12:113.

25. Ha TY, Hwang S, Jung DH, et al. Complications analysis

Cite this article as: Terasaki F, Kaneoka Y, Maeda A, Takayama Y, Fukami Y, Takahashi T, Uji M. The impact of standardized methods of hepatic vein reconstruction with an external iliac vein graft. HepatoBiliary Surg Nutr 2021;10(2):163-171. doi: 10.21037/hbsn.2019.09.18 of polytetrafluoroethylene grafts used for middle hepatic vein reconstruction in living-donor liver transplantation. Transplant Proc 2014;46:845-9.

26. Kubota K, Makuuchi M, Sugawara Y, et al. Reconstruction of the hepatic and portal veins using a patch graft from the right ovarian vein. Am J Surg 1998;176:295-7.

27. Dokmak S, Aussilhou B, Sauvanet A, et al. Parietal Peritoneum as an Autologous Substitute for Venous Reconstruction in Hepatopancreatobiliary Surgery. Ann Surg 2015;262:366-71. 